\title{
Gallstone-ileus: an uncommon presentation of cholelithiasis
}

\author{
S. S. Madawanarachchi, N. N. Wijesundera, T. W. K. G. Indika \\ Teaching hospital Karapitiya, Sri Lanka
}

Keywords: Cholelithiasis; cholecysto-enteric fistula; gallstone ileus; intestinal obstruction

\section{Introduction}

Cholelithiasis is the commonest biliary pathology seen in surgical practice. The majority of patients remain asymptomatic while others present with biliary colics, acute cholecystitis and gallstone pancreatitis. Gallstone ileus is one such uncommon presentation, in which there is a mechanical obstruction of the intestines by an impacted gallstone that has migrated through a bilio-enteric fistula. This condition is seen in elderly patients who have a history of neglected gallstones disease and it carries high morbidity and mortality.

\section{Case presentation}

An 82-year-old female patient presented with a history of feculent vomiting, constipation, progressive abdominal distention and peri-umbilical colicky pain for five days. These symptoms were preceded by bouts of vomiting, nausea and peri-umbilical colicky abdominal pain for 2 months. Furthermore, she had symptoms suggestive of biliary colics for over 20 years for which she hadn't sought medical advice. She was on treatment for hypertension and dyslipidemia.

On admission, she was haemodynamically stable, anicteric and had abdominal distension without signs of peritonitis. Initial conservative management of suspected intestinal obstruction was employed with intravenous fluid resuscitation, intravenous antibiotics and nasogastric decompression. Her blood investigations revealed a neutrophil leucocytosis and an elevated CRP level of 96 $\mathrm{mg} / \mathrm{dl}$. In addition, she had elevated serum creatinine levels suggestive of an acute kidney injury. Imaging with a plain Xray abdomen showed evidence of small intestinal obstruction without an apparent cause. Contrast-enhanced Computed Tomography (CECT) scan was not done due to concerns of renal impairment. Due to progressive worsening of haemodynamic parameters and feculent nasogastric effluent, an exploratory laparotomy was performed with a clinical

Correspondence: Suren Sampath Madawanarachchi

E-mail: kcksuren@gmail.com

(iDhttps://orcid.org/0000-0002-9996-5528

Received: 27-01-2021 Accepted: 21-11-2021 DOI: http://doi.org/10.4038/sljs.v39i3.8791 suspicion of an obstructive lower gastrointestinal malignancy. During laparotomy, small intestinal loops were found to be dilated up to the mid ileum with collapsed distal ileum and large intestines. After adhesiolysis, a cholecysto-duodenal fistula and an impacted gallstone measuring $5 \times 3 \times 2 \mathrm{~cm}$ were found at $10 \mathrm{~cm}$ from the ileocaecal junction causing a distal small intestinal obstruction (Figure 1). The impacted stone was then milked proximally and removed via an enterotomy. No other impacted gallstones were detected. Due to advanced age, co-morbidities and the deteriorated pre-operative general condition of the patient, an intra-operative decision was taken to defer cholecystectomy and repair of the cholecystoduodenal fistula. The patient had an uneventful recovery following the surgery and was discharged on postoperative day five. The follow-up visit at one month revealed a completely asymptomatic patient. The patient refused further surgery to undergo cholecystectomy and cholecystoduodenal fistula repair.

\section{Discussion}

Gallstone ileus is a rare complication of cholelithiasis accounting for $1 \%-3 \%$ of mechanical bowel obstruction. It is seen mostly in elderly women in the seventh to eighth decades of life. This has higher morbidity and mortality owing to other associated co-morbid conditions in these patients [1]. The presence of longstanding gallstones in the gallbladder with resultant chronic inflammation and pressure necrosis may lead to cholecysto-enteric fistulae formation through which gallstones can pass into the gastrointestinal tract. Cholecystoduodenal fistulae are the commonest of these accounting for $75-88 \%$, followed by cholecysto-colonic fistulae [2]. The less common types are cholecysto-gastric, choledocho-duodenal and cholecysto-jejunal fistulae. Stones larger than $2 \mathrm{~cm}$ in diameter can get impacted in the gastro-intestinal tract [1], with ileum being the commonest site of impaction followed by jejunum and duodenum. A stone can get impacted in the sigmoid colon and give rise to large bowel obstruction very rarely. Gallstone ileus may manifest with acute, intermittent or chronic episodes of intestinal obstruction. This patient's history is suggestive of intermittent partial obstruction with waxing and waning of symptoms until the gallstone got fully impacted and produced complete intestinal obstruction. Spontaneous passage of the stones is uncommon once the patient develops symptoms. Pneumobilia dilated small bowel 


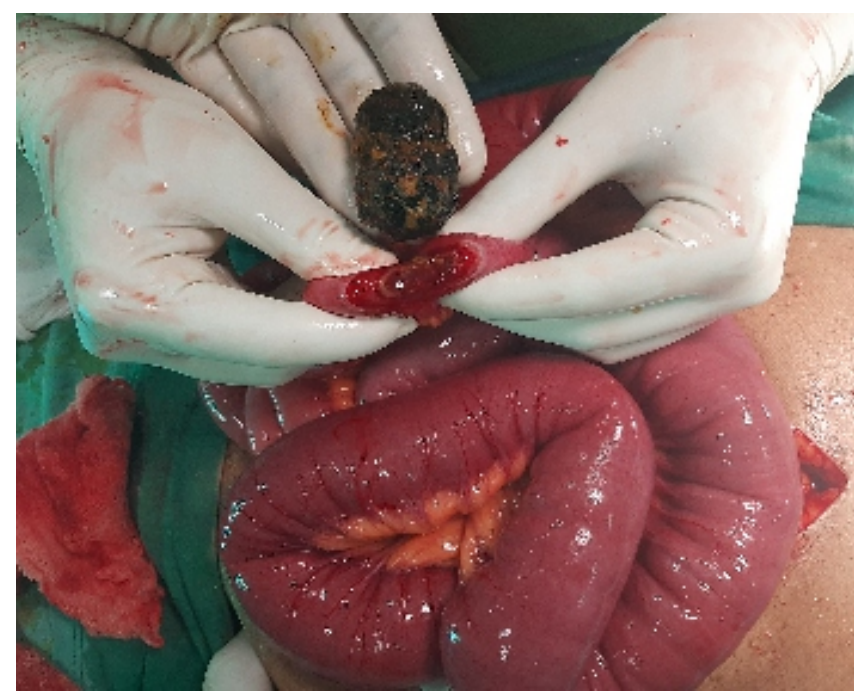

Figure 1. Retrieved Gallstone after enterolithotomy

loops with air-fluid levels and aberrant location of large calcified gallstones are the radiological features seen in the plain X-ray abdomen described as "Rigler's triad". However, only $10 \%$ of the gallstones are sufficiently calcified to be radio-opaque and only $20-35 \%$ of patients with gallstone ileus show the classic triad describe above [1]. CECT would be the best imaging modality with high sensitivity of $93 \%$ to diagnose the condition pre-operatively [3]. However preoperative diagnosis could not be made in this patient due to the absence of pneumobilia in the X-Ray abdomen and the relative contraindication to perform a CECT.

The management of gallstone ileus involves entero-lithotomy to relieve the intestinal obstruction, which is the mainstay of treatment, and subsequent cholecysto-enteric fistula repair and cholecystectomy to prevent recurrences. Latter two steps can be performed at the index laparotomy (one-stage) or a later date (two-stage). Decision on open versus laparoscopic exploration and one-stage versus two-stage procedure is a challenge.

Reisner and Cohen reported a higher mortality rate in the onestage procedure (16.9\%) compared to entero-lithotomy alone (11.7\%) [4]. Marko Doko and colleagues who also compared the two surgical pathways found that although mortality rates were similar, patients who underwent the one-stage procedure had a higher rate of complications [3]. The presence of extensive inflammation and adhesions in and around the right hypochondrium renders it technically difficult to attempt the one-stage procedure leading to longer operative time. Thus enterolithotomy alone is considered the safer option. However, it poses a risk of recurrent gallstone ileus, acute cholecystitis and acute cholangitis while awaiting the next stage of surgery.

\section{Conclusion}

Gallstone ileus is an infrequent entity that is potentially fatal. This should be considered in the differential diagnosis of an elderly patient presenting with intestinal obstruction and a history of neglected gallstones disease. This high degree of clinical suspicion not only enables surgeons to equip themselves with pre-operative imaging but also to plan and stage the surgical intervention. Enterolithotomy alone is the best option for most patients with gallstone ileus. One stage procedure is only justified when the patient's general condition is optimum and intraoperative findings are permissible.

All authors disclose no conflict of interest. The study was conducted in accordance with the ethical standards of the relevant institutional or national ethics committee and the Helsinki Declaration of 1975, as revised in 2000 .

\section{References}

1. Minh B. Luu, Daniel J. Deziel. Unusual complications of Gallstones. Surgical Clinics of North America. 2014 Apr 04; 94(2): 377-394. https://doi.org/10.1016/j.suc.2014.01.002

2. Petrowsky H, Clavien P : Biliary fistula, gallstone ileus, and Mirizzi's syndrome. In: Clavien P, Baillie J (eds) : Diseases of the gallbladder and bile ducts: Diagnosis and treatment. 2nd edition. Malden (MA): Blackwell Publishing; 2008. p. 239-51. https://doi.org/10.1002/9780470986981

3. Doko, M., Zovak, M., Kopljar, M. et al. Comparison of Surgical Treatments of Gallstone Ileus: Preliminary Report. World J. Surg. 27,400-404 (2003). https://doi.org/10.1007/s00268-002-6569-0

4. Reisner RM, Cohen JR. Gallstone ileus: a review of 1001 reported cases. The American Surgeon. 1994 Jun;60(6):441-446.

\section{Learning Points:}

- Gall stone ileus should be considered in the differential diagnosis of elderly patients presenting with intestinal obstruction and a history of neglected gallstone disease

- High degree of clinical suspicion enables the surgeon to be better befitting surgical intervention. 